most buyers knew very little about the products they were buying and were in no way involved in the development stages of new products being designed by their company. As a result, most of the time, the raw materials a buyer had to buy were imposed on him, and he took them under his wing with a certain bitterness of attitude. This created a race of buyers who, knowing nothing about their products and having no choice in their selection, had only one possible outlet for their feelings, and that was by discussing prices in a relatively aggressive way with their suppliers. Such buyers were happy if they reduced the price of a raw material and felt that in doing so they had accomplished something positive for their company. Certainly, this was probably the case short-term, but they had no idea what long-term effects their success would provoke. They had no idea if such a price reduction could be supported by the supplier or if, on the other hand, it was going to make the supplier lose interest in continuing the production of the product in question.

In addition, rather quickly suppliers got the message and would establish their initial prices to take into account the buyer's habits.

In the late 60 s and early 70 s, the importance of better understanding all the implications of negotiating with a supplier started to become apparent. A new generation of buyers began to appear. Buyers with a technical formation which enabled them to better understand and know the products they were buying. This led to buyers looking for a better co-operation with suppliers, but competitivity still was an important factor in everybody's thinking and competitivity still meant low pricing for a lot of people. In fact, the idea of partnerships between suppliers and customers hides an enormous, but fascinating, effort which cannot be accomplished by the buyers alone. They need their colleagues from all the other departments within the organization if they are going to achieve real benefits for their company. It needs everybody to be convinced of the advantages of such a philosophy.

Up to 2 or 3 years ago therefore the situation was more or less as I describe it, with a much more developed idea of creating partnerships between suppliers and customers, even if - to some extent - this was more theoretical and not always put into practice. Everybody was convinced of the theory, but in practice, people continued to say that when there was a problem, it was for the supplier to solve it, whether the problem was one of quality or price. In the last 2 or 3 years, we have seen the development of some very interesting new ideas, such as 'Just in Time', 'SPC' and 'SQC', vendor certification, ISONORM 9000 of the United Nations or British Standard $B S 5750$ or the American Standards $A Q 194,195$, and 196 for example. These are all ideas that can only have a chance of success if co-operation between a supplier and customer is pushed to the very limits. Most of these ideas depend on a customer having the right to carry out an audit in his supplier's premises and that will only be possible if a very open state of co-operation exists between companies. In addition, these new postulations will make it difficult to envisage multiple sourcing. If we want the sort of co-operation between suppliers and customers that 'Just in Time' and vendor certification require, it will be difficult to introduce at the same time the idea of competitors, because the need for competitors is tantamount to saying you are not completely sure about being able to rely on a single source. We will therefore be forced to accept more and more the idea of single sourcing which, until recently, was a sign of insecurity for traditional buyers.

These new theories must be encouraged because it is in everybody's interest to see better co-operation amongst companies, but it will take some time for the mentality of most of us to be adjusted to become compatible with this new era of co-operation. These new theories are going to present many problems and challenges for us, not least the psychological ones. However, they will also give us the chance for many opportunities to carry out our work more effectively and produce improvements in efficiency, quality, and costs. It is a challenge which will represent a big supplementary effort at the beginning, but from which all our companies should be able to benefit in the long-term.

\section{'Make or Buy?' Eine Kernfrage in der Entwicklung neuer Produkte}

\section{Peter Pollak*}

Zusammenfassung. Auch in der chemischen Industrie geht der Trend weg vom 'Alles-selber-Machen', und besonders vor der Aufnahme der Produktion neuer Produkte werden vermehrt 'Make-or-Buy'-Überlegungen angestellt. Für die Favorisierung des 'Buy' gegenüber dem 'Make' gibt es strategische, finanzielle, wirtschaftliche und technische Gründe:

- Priorisierung der finanziellen und menschlichen Ressourcen auf Forschung und Entwicklung und Marketing gegenüber der Produktion.

- Kaum mehr Bedarf für Monoanlagen wegen kürzerer Lebenszyklen und niedrigerer Dosierung bei neuen Pharmazeutika und Pflanzenschutzmitteln.

- Strengere behördliche Auflagen für den Bau von neuen Anlagen und die Vertriebsbewilligung für neue Produkte. Damit grosse Unsicherheiten über den Zeitpunkt der Betriebsaufnahme und die Auslastung der Produktionskapazitäten.

- Vorhandensein/Beherrschen spezieller Technologien bei Dritten.

Schliesslich kommt es mehr und mehr zu einer Arbeitsteilung zwischen rückwärtsintegrierten, industrieorientierten Produzenten von Zwischenprodukten und vorwärtsorientierten, konsumentenorientierten Applikatoren und Vermarktern von Spezialitäten.

\footnotetext{
* Korrespondenz: Dr. P. Pollak, Lonza AG, Postfach, $\mathrm{CH}-4002$ Basel
}

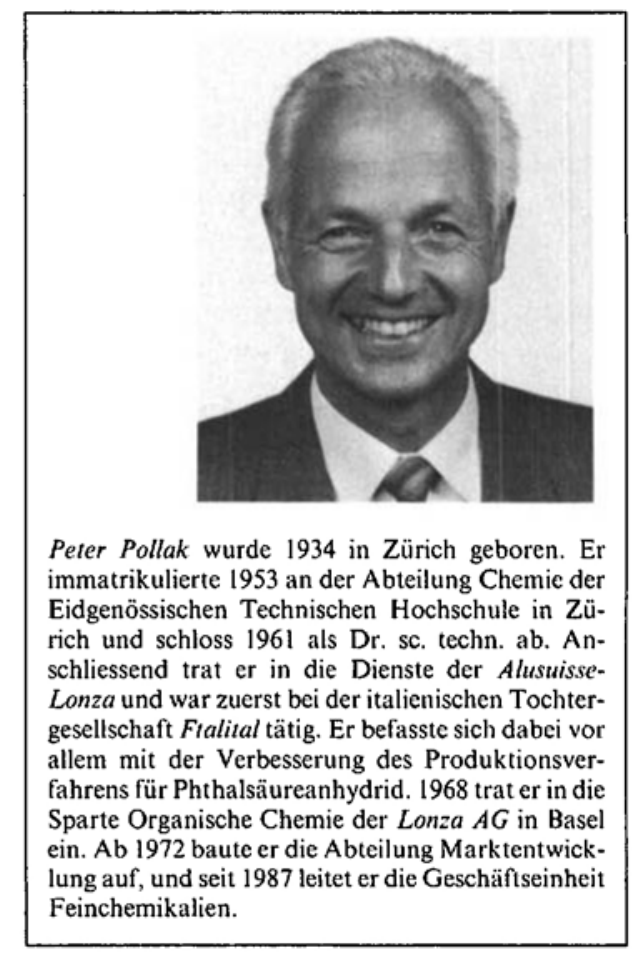

\section{Einleitung}

Auch in der chemischen Industrie geht der Trend weg vom 'Alles-selber-Machen', und vor allem vor der Aufnahme der Pro- 
most buyers knew very little about the products they were buying and were in no way involved in the development stages of new products being designed by their company. As a result, most of the time, the raw materials a buyer had to buy were imposed on him, and he took them under his wing with a certain bitterness of attitude. This created a race of buyers who, knowing nothing about their products and having no choice in their selection, had only one possible outlet for their feelings, and that was by discussing prices in a relatively aggressive way with their suppliers. Such buyers were happy if they reduced the price of a raw material and felt that in doing so they had accomplished something positive for their company. Certainly, this was probably the case short-term, but they had no idea what long-term effects their success would provoke. They had no idea if such a price reduction could be supported by the supplier or if, on the other hand, it was going to make the supplier lose interest in continuing the production of the product in question.

In addition, rather quickly suppliers got the message and would establish their initial prices to take into account the buyer's habits.

In the late 60 s and early 70 s, the importance of better understanding all the implications of negotiating with a supplier started to become apparent. A new generation of buyers began to appear. Buyers with a technical formation which enabled them to better understand and know the products they were buying. This led to buyers looking for a better co-operation with suppliers, but competitivity still was an important factor in everybody's thinking and competitivity still meant low pricing for a lot of people. In fact, the idea of partnerships between suppliers and customers hides an enormous, but fascinating, effort which cannot be accomplished by the buyers alone. They need their colleagues from all the other departments within the organization if they are going to achieve real benefits for their company. It needs everybody to be convinced of the advantages of such a philosophy.

Up to 2 or 3 years ago therefore the situation was more or less as I describe it, with a much more developed idea of creating partnerships between suppliers and customers, even if - to some extent - this was more theoretical and not always put into practice. Everybody was convinced of the theory, but in practice, people continued to say that when there was a problem, it was for the supplier to solve it, whether the problem was one of quality or price. In the last 2 or 3 years, we have seen the development of some very interesting new ideas, such as 'Just in Time', 'SPC' and 'SQC', vendor certification, ISONORM 9000 of the United Nations or British Standard $B S 5750$ or the American Standards $A Q 194,195$, and 196 for example. These are all ideas that can only have a chance of success if co-operation between a supplier and customer is pushed to the very limits. Most of these ideas depend on a customer having the right to carry out an audit in his supplier's premises and that will only be possible if a very open state of co-operation exists between companies. In addition, these new postulations will make it difficult to envisage multiple sourcing. If we want the sort of co-operation between suppliers and customers that 'Just in Time' and vendor certification require, it will be difficult to introduce at the same time the idea of competitors, because the need for competitors is tantamount to saying you are not completely sure about being able to rely on a single source. We will therefore be forced to accept more and more the idea of single sourcing which, until recently, was a sign of insecurity for traditional buyers.

These new theories must be encouraged because it is in everybody's interest to see better co-operation amongst companies, but it will take some time for the mentality of most of us to be adjusted to become compatible with this new era of co-operation. These new theories are going to present many problems and challenges for us, not least the psychological ones. However, they will also give us the chance for many opportunities to carry out our work more effectively and produce improvements in efficiency, quality, and costs. It is a challenge which will represent a big supplementary effort at the beginning, but from which all our companies should be able to benefit in the long-term.

\section{'Make or Buy?' Eine Kernfrage in der Entwicklung neuer Produkte}

\section{Peter Pollak*}

Zusammenfassung. Auch in der chemischen Industrie geht der Trend weg vom 'Alles-selber-Machen', und besonders vor der Aufnahme der Produktion neuer Produkte werden vermehrt 'Make-or-Buy'-Überlegungen angestellt. Für die Favorisierung des 'Buy' gegenüber dem 'Make' gibt es strategische, finanzielle, wirtschaftliche und technische Gründe:

- Priorisierung der finanziellen und menschlichen Ressourcen auf Forschung und Entwicklung und Marketing gegenüber der Produktion.

- Kaum mehr Bedarf für Monoanlagen wegen kürzerer Lebenszyklen und niedrigerer Dosierung bei neuen Pharmazeutika und Pflanzenschutzmitteln.

- Strengere behördliche Auflagen für den Bau von neuen Anlagen und die Vertriebsbewilligung für neue Produkte. Damit grosse Unsicherheiten über den Zeitpunkt der Betriebsaufnahme und die Auslastung der Produktionskapazitäten.

- Vorhandensein/Beherrschen spezieller Technologien bei Dritten.

Schliesslich kommt es mehr und mehr zu einer Arbeitsteilung zwischen rückwärtsintegrierten, industrieorientierten Produzenten von Zwischenprodukten und vorwärtsorientierten, konsumentenorientierten Applikatoren und Vermarktern von Spezialitäten.

\footnotetext{
* Korrespondenz: Dr. P. Pollak, Lonza AG, Postfach, $\mathrm{CH}-4002$ Basel
}

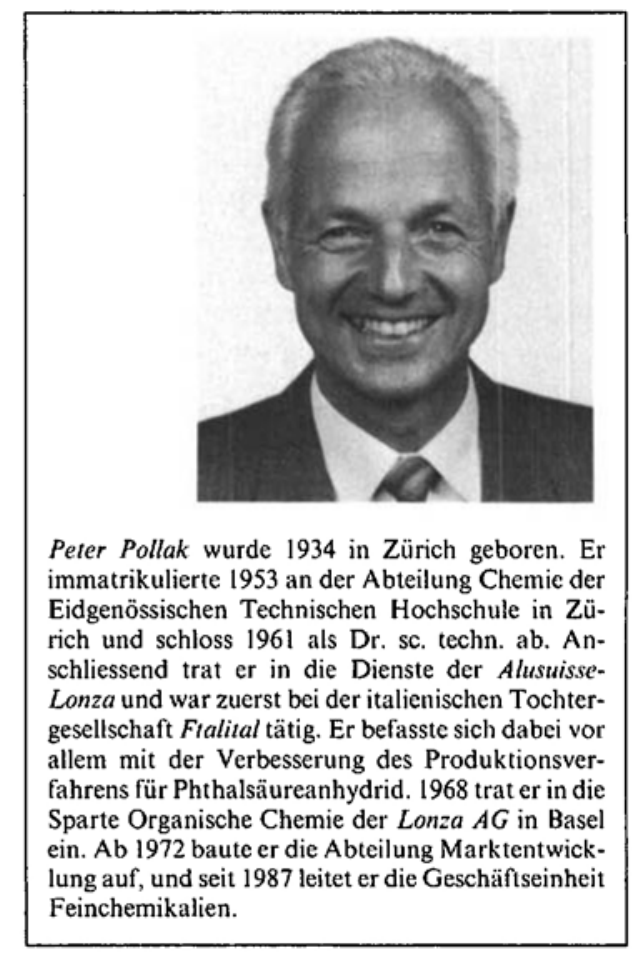

\section{Einleitung}

Auch in der chemischen Industrie geht der Trend weg vom 'Alles-selber-Machen', und vor allem vor der Aufnahme der Pro- 


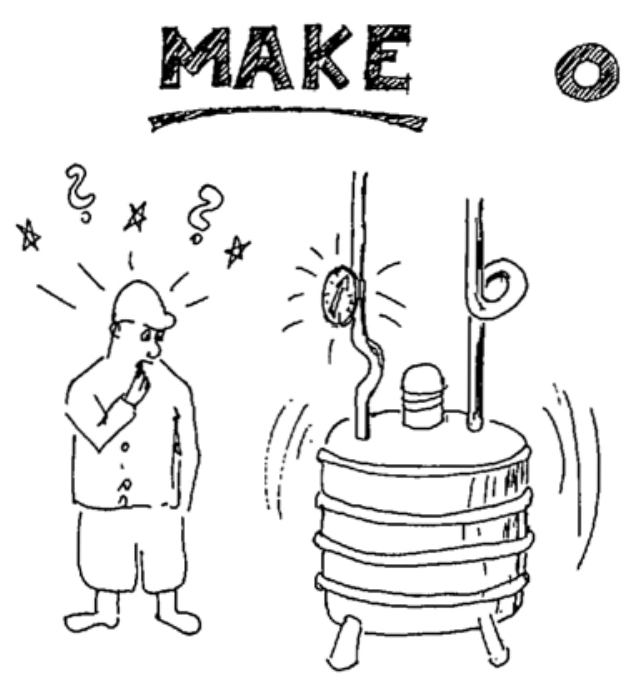

Fig. I

\begin{tabular}{|lcr|}
\hline mature & product life cycle position & \\
\hline & manufacturing plant & \\
existing & & nom existing \\
\hline & technology & new \\
\hline known & raw materials & not \\
\hline $\begin{array}{l}\text { readily } \\
\text { available }\end{array}$ & & available \\
\hline & & \\
\hline
\end{tabular}

Fig. 2. Make or buy ... and product life cycle

duktion neuer Produkte werden vermehrt 'Make-or-Buy'-Überlegungen angestellt (Fig. 1). Wie bereits aus dem Untertitel des Referats hervorgeht, muss man zum voraus zwei Fälle klar unterscheiden, nämlich

- Produktion von bestehenden Produkten, die den Zenith der Lebenskurve erreicht haben, in bestehenden Anlagen mit vertrauten Technologien,

- Produktion von neuen Produkten in der Anfangsphase der Lebenskurve, für die keine Anlagen bestehen und wo die zukünftigen Bedarfsmengen noch sehr schwer abzuschätzen sind (Fig. 2).

Es steht ausser Frage, dass im ersten Fall die Eigenherstellung im Vordergrund steht. Im zweiten Fall sprechen gewichtige Gründe für das 'Buy instead of Make'. Es gibt kommerzielle, technische, finanzielle und strategische Überlegungen, die bei einem 'Make-or-Buy'-Entscheid zu berücksichtigen sind. Im folgenden sollen diese vier Gesichtspunkte der Reihe nach diskutiert werden.

\section{Kommerzielle Aspekte}

Betrachten wir zunächst das folgende Szenario: Ein Chemieunternehmen, das vor allem im Bereich Agrochemikalien und Pharmazeutika tätig ist, hat einen neuen Wirkstoff entdeckt. Der Wirkstoff hat das erste Screening und in-vitro-Tests erfolgreich bestanden. Die beteiligten Forscher sind von dem Projekt sehr eingenommen, und die Begeisterung nimmt zu. Für die Herstellung der ersten paar hundert Gramm der neuen Chemikalie wurde eine provisorische Laborvorschrift entwickelt. Das Ziel war dabei, Material für erste präklinische bzw. Gewächshausstudien bereitzustellen. Irgendwelche prozessbezogene Fragen wie Verfügbarkeit von Rohmaterialien, Anzahl Verfahrensschritte, Ausbeute, Produktivität, Sicherheit und Umwelt wurden noch nicht untersucht. In der Praxis bedeutet das, dass jetzt die Verfahrensentwicklung einsetzen sollte. Mit anderen Worten, die erste 'Make-or-Buy'Entscheidung steht an. Die Antwort wird in erster Linie davon abhängen, in welchem Mass eigene menschliche und technische Ressourcen vorhanden sind. Wenn aus der Erprobung der neuen Substanz weiterhin positive Resultate anfallen, wird sich früher oder später die Frage der industriellen Produktion stellen. Um die vom Marketing prognostizierten Mengen produzieren zu können, muss die Grösse der Anlage für den neuen Renner spätestens zwei Jahre vor der Markteinführung bestimmt werden. In dieser relativ frühen Entwicklungsphase ist eine einigermassen verlässliche Bedarfsprognose sehr schwie- rig. Zur Illustration wollen wir einmal nur drei Parameter, die einen wesentlichen Einfluss auf das zukünftige Absatzvolumen haben, etwas näher betrachten, nämlich die Anzahl Patienten resp. die landwirtschaftliche Nutzfläche, den Marktanteil und die Anwendungsdosis in mg pro Tag pro Patient resp. in $\mathrm{g}$ pro ha pro Saison (Tab. 1). Als praktisches Beispiel sei das Haarwuchsmittel 'Minoxidil' angeführt. Jeder dieser drei Faktoren kann in einem weiten Bereich variieren. Es genügt die Frage zu stellen, wieviel Männer welche Wirkstoffmenge dieser Lotion wieviel Mal pro Tag wie lange anwenden werden. Bei einer Schwankungsbreite von 1:2 pro Faktor beträgt die gesamte Variationsbreite $1^{3}: 2^{3}$, d.h. $1: 8$.

\section{Technische Aspekte}

Die Dimensionierung der geplanten Anlage wird nicht nur durch die vorgenannten marktrelevanten Faktoren, sondern auch durch technische Gegebenheiten beeinflusst. Zunächst hängt die Art und Grösse der zu bauenden Produktionsanlage natürlich sehr stark davon ab, welcher Prozess gewählt wird. Im Fall von Pyrimidinen, Schlüssel-Zwischenprodukten für viele Pharmazeutika und Pflanzenschutzmittel, existieren drei grundsätzlich verschiedene Herstellungsmethoden, nämlich die Verknüpfung eines $\mathrm{N}-\mathrm{C}-\mathrm{N}$ - mit einem $\mathrm{C}-\mathrm{C}-\mathrm{C}-$ Fragment, die Verknüpfung eines $\mathrm{C}-\mathrm{C}-\mathrm{C}-\mathrm{N}$ - mit einem $\mathrm{C}-\mathrm{N}-\mathrm{Frag}-$ ment und schliesslich die Reaktion eines $\mathrm{N}-\mathrm{C}-\mathrm{C}-\mathrm{C}-\mathrm{N}-\mathrm{Fragmentes}$ - typisch Malonsäuredinitril - mit einem einzigen $\mathrm{C}$ Atom (Fig. 3). Je nach der Art des oder der Substituenten ist eine andere Methode am besten geeignet (vgl. Lonza-Broschüre: Pyrimidines, Pteridines and Purines, von Prof. Dr. h.c. W. Pfleiderer, Basel, 1986).

Die Situation wird noch komplizierter, wenn spezifische Technologien benötigt werden, wie z. B. hoher Druck, tiefe Temperatur oder sensitive Rohstoffe wie Blausäure, Mercaptane, Ozon, Phosgen. Wenn

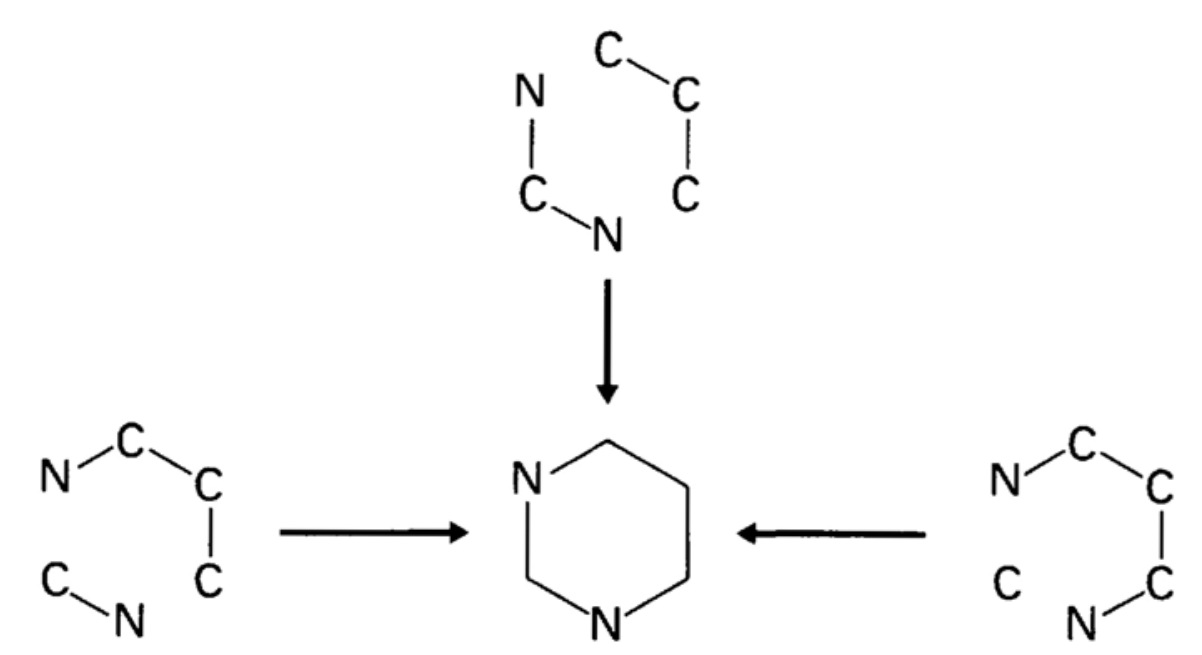

Fig. 3. Pyrimidine synthesis. The principal synthetic method. 


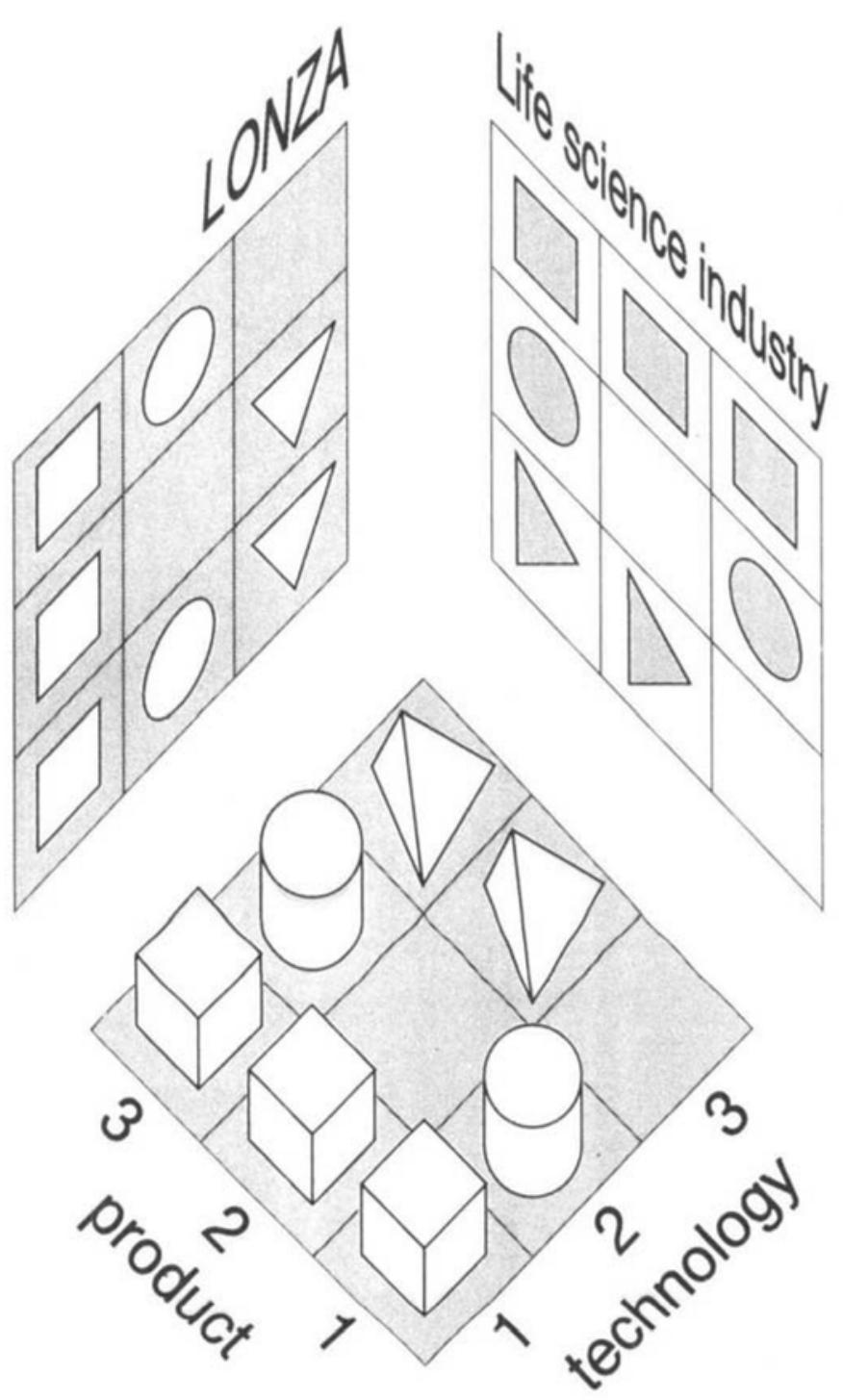

Fig. 4. Product / Technology matrix
- Reaktionszeiten

Erfahrene Verfahrensingenieure horchen auf, wenn in einer Herstellungsvorschrift geschrieben steht ' 15 Stunden am Rückfluss kochen'. Diese Zeitangabe stammt normalerweise daher, dass der Laborchemiker abends um 5 Uhr aufhört und morgens um 8 wieder zu arbeiten beginnt. Über Nacht lässt er 'rückflussieren'...

\section{- Konzentration}

Der Laborchemiker schenkt der Konzentration des gewünschten Produktes in der Reaktionslösung normalerweise wenig Beachtung. Sie kann aber sehr wichtig sein, nicht nur für die Festlegung der Reaktorgrösse, sondern auch für die Auslegung von Hilfsapparaturen, wie z.B. Lösungsmittel-Rückgewinnungsanlagen.

\section{- Umwelt}

Die sichere Entsorgung von Nebenprodukten und Abfallströmen kann den gleichen apparativen Aufwand benötigen, wie das eigentliche Herstellungsverfahren.

Die vorgenannten Faktoren bestimmen die Produktionsleistung pro Reaktorvolumeneinheit, welche das Schlüsselelement für die Dimensionierung der Anlage und der Produktionskosten darstellt.

Alles in allem hat eine Pharma- oder Pestizidfirma, die die Herstellung einer neuen Spezialität plant, über eine recht bedeutende Investition zu entscheiden heutzutage typisch eine zwei- bis dreistellige Zahl von Millionen Franken -, bevor eine solche Technologie bzw. solche Chemikalien nur für einen einzelnen Verfahrensschritt und ein einzelnes spezifisches Endprodukt benötigt werden, ist es gewöhnlich lohnender, die Synthese bei einer Chemiefirma durchführen zu lassen, welche über das entsprechende Know-how und die entsprechenden Anlagen verfügt.

Fig. 4 illustriert die Situation aus der Sicht des Abnehmers und des Anbieters: Ersterer benötigt kleine Kapazitäten verschiedener Technologien für sein neues Pflanzenschutzmittel oder Pharmazeutikum. Jede für sich liegt unterhalb der minimum economical size für eine Neuanlage. Letzterer ist in der Lage, spezifische Technologien mehreren Kunden anzubieten. Durch die Kombination mehrerer Projekte, die die gleichen Technologien benötigen, gelangt er zu einer vernünftigen Auslastung seiner spezifischen Anlage.

Selbst wenn der Prozess festgelegt ist, gibt es immer noch verschiedene Faktoren, welche die Grösse der zukünftigen Anlage beeinflussen und die zum derzeitigen Zeitpunkt noch nicht bekannt sind, wie z.B.

- Gesamtausbeute

Während der Verfahrensentwicklung sind oft bedeutende Ausbeuteverbesserungen möglich. metric tons

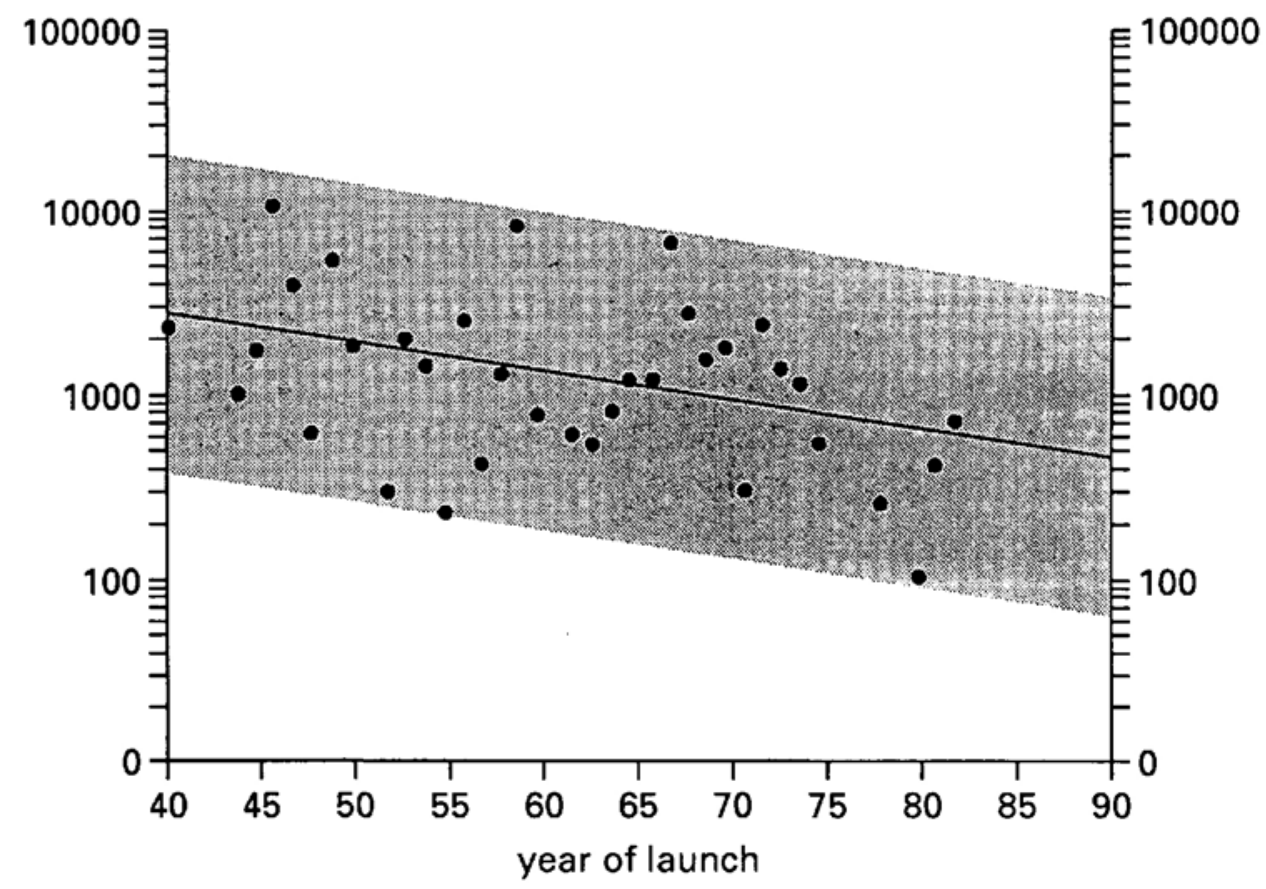

Fig. 5. US pesticide consumption 
Tab.1. Factors Affecting Demand for New Drugs/Pesticides

\begin{tabular}{llcc}
\hline Drugs & Pesticides & Level \\
\hline 1 Number of patients & Total crop acrage & 1 & 2 \\
2 Market share & Market share & 1 & 2 \\
3 Daily dose & Application rate & 1 & 2 \\
\hline Total & & $=1^{3}: 2^{3}$ \\
& & $=1$ & $: 8$ \\
\hline
\end{tabular}

das Marketing in der Lage ist, eine zuverlässige Bedarfsprognose abzugeben, bevor die F \& E ein optimiertes Herstellungsverfahren ausgearbeitet hat und bevor die Ingenieurabteilung weiss, was sie eigentlich bauen muss ... Die Situation ist für solche Firmen besonders kritisch, welche nicht gleichzeitig eine Mehrzahl von neuen Produkten in der Entwicklung haben, die sich für die Herstellung in der gleichen Mehrprodukteanlage eignen, und das ist heute fast der Normalfall.

Impliziert ist dabei die wichtige Erkenntnis, dass heute in aller Regel die Aktivität neuer Pharma- oder PflanzenschutzmittelSpezialitäten so hoch ist, dass die benötigten Mengen den Bau von Monoanlagen nicht mehr gestatten. Dies ist sehr schön an der Entwicklung der Produktionszahlen von Pflanzenschutzmitteln im Verlauf der letzten 20 Jahre zu verfolgen (Fig. 5): So lag der Ausstoss von 'Triazin'-Herbiziden in den 60er Jahren in der Grössenordnung von mehreren 10000 Tonnen, in den 70er Jahren für Produkte im gleichen Anwendungsgebiet im Grössenbereich von 10000 Tonnen - vgl. die 'Amid'-Herbizide - und bei den neuesten 'Sulfonylharnstoff'-Herbiziden liegt die produzierte Jahresmenge in der Grössenordnung von einigen 100 Tonnen.

\section{Finanzielle Aspekte}

Der Bau einer neuen Anlage zu einem Zeitpunkt, da über den Markterfolg des darin herzustellenden Produktes notwendigerweise noch nichts bekannt ist, ist auch ein finanzielles Risiko. Beim 'Buy instead of make' muss man erst dann zahlen, wenn man auch verdient. Auf amerikanisch 'Pay

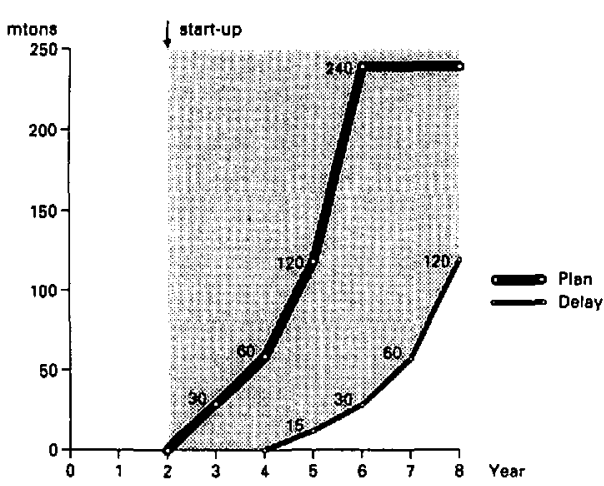

Fig. 6. Project USA: demand forecast as you earn'. Mit anderen Worten: es muss nicht viele Jahre, bevor man mit der Markteinführung einer neuen Spezialität beginnt, Geld in den Bau von Produktionsanlagen investiert werden, mit dem Risiko, dass die Anlage niemals gebraucht wird.

Diesem Vorteil steht der Nachteil gegenüber, dass der Lohnhersteller ebenfalls Geld verdienen will; Geld, das der Abnehmer lieber in der eigenen Firmenkasse sähe.

Anhand eines vereinfachten Rechenbeispiels soll jetzt gezeigt werden, dass es aus finanzieller Sicht eine sehr weise Entscheidung sein kann, keine neue Anlage für das neue Produkt zu bauen, sondern mit einem Partner zusammenzuarbeiten, der über Mehrprodukte-Kapazitäten verfügt. Von welchen Prämissen gehen wir aus? Wir sind jetzt Ende März 1990, und aufgrund nicht allzu vertrauenerweckender Zahlen vom Marketing soll die Anlage für eine Kapazität von 240 jato geplant werden. Diese Anlage soll in zwei Jahren, d.h. am 1. April 1992 in Betrieb gehen. Wenn alles nach Plan geht, d.h. wenn bei den klinischen Versuchen oder bei den Feldversuchen keine unerwünschten Nebenwirkungen auftreten und wenn die FDA oder EPA rechtzeitig die Zulassung erteilen, und wenn die Konkurrenz nicht in der Zwischenzeit mit einem ähnlichen Produkt auf den Markt kommt, wenn alle diese Wenn's zutreffen, wird sich der Bedarf so entwickeln, wie in der dicken Kurve auf der Fig. 6 aufgetragen. Da der anvisierte Umsatz erst nach einer Anlaufphase von etwa zwei Jahren erreicht werden wird, bestehen in den Jahren 1992 und 1993 erhebliche freie Kapazitäten. Diese Leerkapazitäten müssen selbstverständlich in der einen oder anderen Weise bezahlt werden. Die Dinge sehen viel schlechter aus, wenn die behördlichen Zulassungen verzögert werden, wenn ein Konkurrent Marktanteile gewinnt oder wenn das Applikationsfeld eingeschränkt werden muss. Das kann ohne weiteres bedeuten, dass 1995 anstelle von 240 Tonnen nur 120 Tonnen produziert werden oder sogar nur 30 Tonnen, wenn die eingangs erwähnte 1:8-Regel Anwendung findet! Eine leider nicht allzu selten auftretende Situation. Im letzteren Fall bedeutet die ganze dunkel schraffierte Fläche Leerkapazität resp. nicht rekuperierte Fixkosten. Was bedeutet das in Franken und Rappen?

Um die Vorteile des 'Buy' gegenüber dem 'Make' zu illustrieren, haben wir einen typischen Fall durchgerechnet für einen Wirkstoff, den unsere Firma für eine amerikanische Pharmafirma herstellt. Die Grundannahmen sind in Tab. 2 dargestellt. Wir nehmen an, dass die Herstellungskosten bei unserem Kunden und bei uns etwa dieselben sind, und wir gehen weiter davon aus, dass die amerikanische Firma 80 Mio. $\$$ in eine Neuanlage investieren muss, wohingegen wir eine bestehende Anlage für 10 Mio. für diese spezifische Produktion umrüsten können. Wir werden einen Verkaufspreis von $350 \$ / \mathrm{kg}$ verlangen. Die Bruttomarge von $100 \$ / \mathrm{kg}$ wird es erlauben, die Anpassungsinvestition zu amortisieren und einen vernünftigen Ertrag zu erwirtschaften

In Fig. $7 a$ sind 2 'Make'-Situationen dargestellt:

1. Das Projekt entwickelt sich plangemäss. Wenn man die dicke, gestrichelte Linie von links oben verfolgt, dann erreicht man nach zwei Baujahren den Punkt, wo 80 Mio. \$ für den Bau der Anlage ausgegeben worden sind. Im Jahr 3 werden 30 Tonnen zu $250 \$ / \mathrm{kg}$ hergestellt, was das Projekt mit weiteren 7,5 Mio. belastet, usw., bis zu Ende des Jahres 8, wenn total 930 Tonnen produziert sein werden, zu Gesamtkosten von 236,5 Mio. \$.

2. Wenn das Projekt verzögert ist, werden natürlich die 80 Mio. \$ zu Anfang des Jahres 3 ebenfalls ausgegeben worden sein. Da aber die total zwischen den Jahren 3 und 8 produzierte Menge nur 225 Tonnen beträgt, wird die Gesamtausgabe nur 136 Mio. \$ betragen.

Tab.2. Project USA: Investment, Cost, and Price Assumptions

\begin{tabular}{lcc}
\hline & US Drug Company & Fine Chemical Manufacturer \\
\hline Investment (MM \$) & 80.00 & $\left.10.00^{1}\right)$ \\
Manufacturing cost $\left(\$ / \mathrm{kg}^{2}\right)$ & 250.00 & 250.00 \\
Sales price (\$kg) & - & 350.00 \\
Penalty & - & $15 \%$ of difference plandelay \\
& & plus amortisation of adapta- \\
& & tion cost \\
\hline
\end{tabular}

1) Adaptation of existing Multy Purpose Plant.

$\left.{ }^{2}\right)$ Full cost including raw materials, labor, maintenance, plant and general overhead, amortisation.

No allowance made for additional cost in case of smaller production in the first years. 
a)

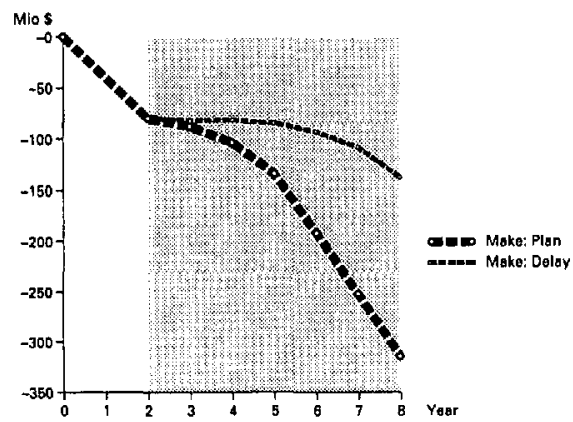

Fig. 7. a) Project USA: total expenses/make. b) Project USA: total expenses/make or buy

In Fig. $7 b$ ist die 'Buy'-Situation darüber gelegt: Da der Lohnhersteller die Anpassungskosten selber trägt, bestehen vor dem Jahr 3 keinerlei Ausgaben (vgl. die dicke Linie in Fig. 7b). Da der Abnehmer aber $100 \$ / \mathrm{kg}$ mehr pro kg gekauftes Material zahlt, nimmt der Kostenvorteil gegenüber dem Selbermachen im Laufe der Zeit ab. Wenn das Projekt nach Plan geht, wird der 'Breakeven point' kurz vor Ende des Jahres 8 erreicht - das ist der Preis, der für die

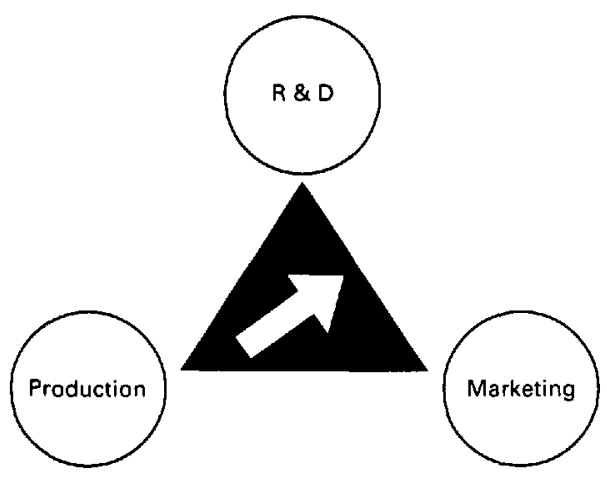

Fig. 8. Shift in investment priorities

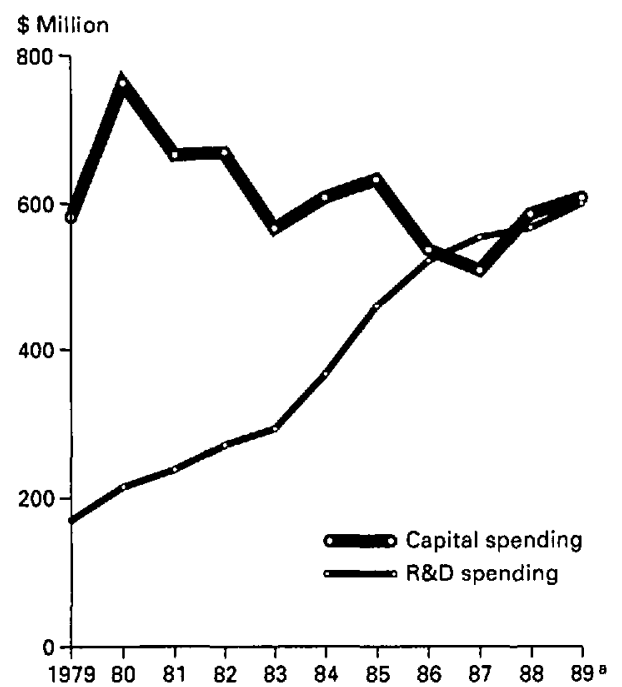

a) Estimate .

Fig. 9. $R \& D$ spending has surged to capital spending levels (example: MONSANTO) zolgewinnung Herstellung von Soda usw. zurückführen. Im Einklang mit dieser starken Produktionsorientierung waren auch die obersten Firmenchefs bis vor wenigen Jahren stets Chemiker. Erst in der jüngsten Zeit beginnt sich das Blatt zu wenden....

Ein Ereignis, das diese Wende sehr deutlich dokumentiert, ist die Aufgabe der Eigenherstellung von Methanol durch die $B A S F$. Ganz anders ist die Situation in den USA, wo grosse Pharmaunternehmen aus Drugstores hervorgegangen sind und Marketing immer noch im Vordergrund der unternehmerischen Tätigkeit steht. b) Mios

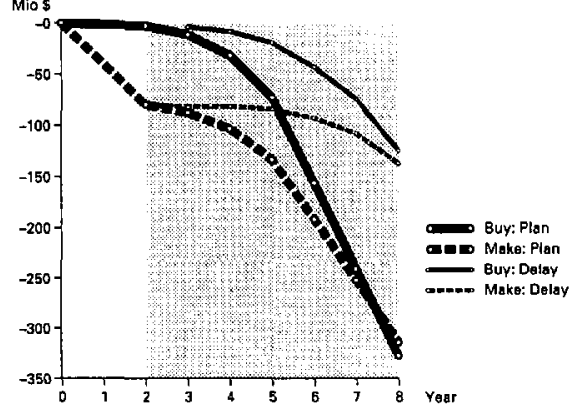

Wenn wir uns jetzt von der Vergangenheit ab- und der Gegenwart zuwenden, so stellen wir fest, dass der optimale Einsatz menschlicher, technischer und finanzieller Ressourcen für den Erfolg eines Unternehmens entscheidend geworden ist. Selbst sehr profitable Unternehmen müssen in der Allokation ihrer Mittel Prioritäten setzen. Und in der chemischen Industrie geht hier der Trend eindeutig in Richtung auf Ausbau von Forschung und Entwicklung und Marketing und weniger Produktion (Fig. 8).

Illustrativ ist die Entwicklung bei $M O N$ $S A N T O$, einem der 20 grössten Chemieunternehmen der Welt (vgl. Fig. 9). Während die Investitionen im Laufe der letzten Jahre abnehmende Tendenz zeigen, nehmen die Ausgaben für Forschung und Entwicklung laufend zu. 1986 haben die F \& E-Ausgaben erstmals die Ausgaben für Investitionen überstiegen. Tatsache ist, dass MONSANTO die wichtigsten Vorprodukte für seine beiden grössten Pflanzenschutzmittel, Lasso und Round-up, nicht selbst herstellt. Zu berücksichtigen ist dabei noch, dass heute von den Investitionen ein immer kleinerer Teil effektiv auf Produktionsanlagen entfällt. Die Aufwendungen für Infrastruktur, Sicherheit, Entsorgung beanspruchen immer mehr Platz.

Schliesslich spielen auch noch der Stellenwert und die organisatorische Eingliederung der Einkaufsabteilung eine Rolle. Wenn in der Firmenkultur der Einkauf die Bedeutung eines 'Bestellungen-Schreibbüros' hat, ist er schlecht für moderne Beschaffungsmethoden gerüstet. Im Rahmen des 'Material Managements' kommt ihm heute vielmehr die Aufgabe zu, in enger Zusammenarbeit mit Entwicklungs- und Produktionsabteilungen zu ermitteln,

- auf welcher Stufe ein Vorprodukt einer neuen Spezialität beschafft werden soll

- welche oder, im Rahmen des single sourcing, welcher Lieferant mit der Herstellung einer ganz bestimmten, bisher kommerziell nicht erhältlichen Chemikalie betraut werden soll.

Diese Aufgaben können nur im Rahmen von Teams, welche Vertreter von kommerziellen und technischen Stellen des Kunden und der eigenen Firma umfassen, gelöst werden.

\section{Schlusswort}

'Make or Buy', global sourcing, single sourcing sind Unternehmenskonzepte, die zuerst in der Maschinenindustrie entwikkelt worden sind. Sie gewinnen aber auch in der chemischen Industrie zunehmend an Bedeutung, und es zeichnet sich eine Arbeitsteilung ab zwischen rückwärtsintegrierten, industrieorientierten Produzenten von Zwischenprodukten und vorwärtsintegrierten, konsumentenorientierten Applikatoren und Vermarktern von Spezialitäten. 